\title{
Tissue Inhibitor of Metalloproteinases-2 Mediates Kidney Injury during Sepsis
}

\author{
Xiaoyan Wen Jicheng Zhang Xiaojian Wan Alicia A. Frank Xin Qu \\ John A. Kellum \\ Department of Critical Care Medicine, Center for Critical Care Nephrology, University of Pittsburgh School of \\ Medicine, Pittsburgh, PA, USA
}

\section{Keywords}

Secretory TIMP-2 $\cdot$ Kidney injury $\cdot$ Sepsis

\begin{abstract}
Introduction: Urinary tissue inhibitor of metalloproteinases (TIMP)-2 has been identified as a predictive marker for acute kidney injury (AKI), including sepsis-associated AKI (S-AKI). Whether TIMP-2 might be causally related to AKI and hence represent a viable drug target is unclear. Objective: The aim of this study was to evaluate whether suppression of TIMP-2 attenuates S-AKI. Methods: Balb/c mice were randomized to sham or cecal ligation and puncture surgery and treated with or without a TIMP-2-neutralizing antibody. Animals were followed for $48 \mathrm{~h}$ and then sacrificed for analysis of TIMP-2 expression, cell cycle, and histology. Results: AntiTIMP-2 resulted in decreased lumen TIMP-2 expression
\end{abstract}

Xiaoyan Wen and Jicheng Zhang contributed equally.

Contribution from the AKI and CRRT 2020 Symposium at the 25th International Conference on Advances in Critical Care Nephrology, Manchester Grand Hyatt, San Diego, CA, USA, February 24-27, 2020. This symposium was supported in part by the NIDDK-funded University of Alabama at Birmingham-University of California San Diego O'Brien Center for Acute Kidney Injury Research (P30DK079337). which markedly increased cell cycle progression and attenuated epithelial cell injury by histology. Conclusions: TIMP-2 mediates S-AKI and appears to be a viable drug target.

(c) 2020 S. Karger AG, Basel

\section{Introduction}

Acute kidney injury (AKI) is recognized as a common disorder in hospitalized patients and especially in the critically ill and injured. The occurrence of AKI is strongly associated with mortality and excess healthcare costs [1]. Limitations inherent in traditional markers of AKI such as serum $\mathrm{Cr}$ and urinary output have prompted the development of novel diagnostics [2]. In addition, sub-phenotypes of AKI that respond differently to the same treatment $[3,4]$ cannot be differentiated on the basis of serum $\mathrm{Cr}$ or other clinical features. Therefore, new ways of identifying and classifying AKI are urgently needed.

Tissue inhibitor of metalloproteinases (TIMP)-2 was identified as an early biomarker for AKI [5] and has become part of the only FDA-approved test. Use of this test has been shown to reduce development of AKI when tied to a treatment bundle based on the KDIGO AKI guideline [6]. However, little is known about whether TIMP-2 is 
only a marker of AKI or whether it is in the causal pathway for AKI. We [7] have reported rapid release of TIMP from distal tubule epithelial cells in response to various insults, and others $[8,9]$ have shown similar results for urine levels in animal models. TIMP-2 is known to mediate cell cycle arrest in some cell types [10], and this mechanism is believed to be protective in the case of AKI [5, 11]. Thus, blocking TIMP-2 should be harmful. However, $\mathrm{Li}$ and coworkers found that downregulation of TIMP-2 showed reno-protective effects on endotoxin-induced AKI, which was associated with the anti-inflammatory activity through inhibition of the nuclear factor (NF) $-\kappa B$ pathway [9]. These findings suggest a pathogenic role for TIMP-2 in AKI and demonstrate a novel target for the treatment of AKI. Therefore, in this brief communication, we sought to determine if the application of TIMP2-neutralizing antibody could alter cell cycle progression in renal tubular epithelial cells and attenuate histologic damage in sepsis-associated AKI (S-AKI).

\section{Materials and Methods}

\section{Study Design}

$\mathrm{Balb} / \mathrm{c}$ mice were housed, and the experiment was conducted according to the corresponding guidelines. Animals were randomized to sham or cecal ligation and puncture (CLP, 50\% mortality model) surgery; with TIMP-2 antibody $\left(5 \mathrm{mg} \mathrm{kg}{ }^{-1}\right.$, intraperitoneal injection, Astute Medical) or nonspecific immunoglobulin G2b (antibodies online \# ABIN1344452) given immediately following surgery. Animals were given a 1-time fluid resuscitation (warmed saline $40 \mathrm{~mL} \mathrm{~kg}^{-1}$ ) following surgery and buprenorphine $0.05 \mathrm{mg} \mathrm{kg}^{-1} \mathrm{q} 12 \mathrm{~h}$, and ampicillin sodium and sulbactam sodium $300 \mathrm{mg} \mathrm{kg}^{-1} \mathrm{q} 12 \mathrm{~h}$ were administered for 2 days. Animals were followed up to $48 \mathrm{~h}$ and sacrificed at the end point for measurements. To label cells undergoing cell cycle, mice were injected with bromodeoxyuridine (BrdU, Sigma \#B5002) DPBS (10 mg mL $\mathrm{mL}^{-1}$ ) intraperitoneally at a dosage of $150 \mathrm{mg} \mathrm{kg}^{-1} 2 \mathrm{~h}$ prior to euthanizing. Cr levels at the end point were measured (Sigma \#MAK080) to identify kidney dysfunction.

\section{Detection of Protein Expression and Renal Histologic}

Assessment

For Western blotting, kidney tissue samples were homogenized in RIPA lysis buffer (Thermo Fisher Scientific \#89901) and supplemented with a protease/phosphatase inhibitor tablet (Thermo Fisher Scientific \#88668, 1 tablet per $10 \mathrm{~mL}$ ), PMSF (Sigma $\# 10837091001,100 \mu \mathrm{g} / \mathrm{mL}$ ). The protein concentration was determined using Pierce BCA Protein Assay Kit (Thermo Fisher Scientific \#23225) and 20-30 $\mu$ g of protein from lysates was loaded in 4-20\% Tris-Glycine gel (Invitrogen \#EC6028BOX) and separated by electrophoresis. Proteins were then blotted with a primary antibody (anti-TIMP-2, Santa Cruz\#sc-6835; anti-Actin, Santa Cruz\#sc-1616) and then IRDye secondary antibodies (1:5,000, LI-COR) for visualizing using Odyssey Infrared Imaging system (LI-COR).

TIMP-2 Mediates Kidney Injury
For immune staining, the tissue was fixed in $4 \%$ paraformaldehyde and 70\% ETOH, embedded in paraffin, and stained using hematoxylin and eosin (H\&E), or in $2 \%$ paraformaldehyde-PBS and $30 \%$ sucrose and embedded in OCT compound (Fisher scientific) for immunofluorescence. Sliced tissues were stained with anti-TIMP-2 (Cell signal \#5738), anti-CD326 (Thermofisher \#115791-82), anti-CD45 (Thermofisher \#14-0451-81), anti-cyclin E1 (proteintech \#11554), anti-cyclin B1 (Cell signal \#4138), and then corresponding fluorophore-conjugated secondary antibodies (Jackson ImmunoResearch laboratories) and FITC-lotus tetragonolobus lectin (LTL) (Vector laboratories, FL-1321) were mounted with Fluoro-gel II with DAPI (Electron Microscopy Sciences \#17985-50). The H\&E-stained slices were used for assessing kidney histology injury. Semiquantitative renal histology injury scoring was assessed by a renal pathologist blinded to group assignment on a score of $0-5$ according to the percentage area of tubules that exhibit cell necrosis or vacuolization, cast formation, and/or dilation. Both H\&E and fluorescence images were processed using ImageJ.

\section{Preparation of Single-Cell Suspensions and Flow Cytometry}

Assays

Kidneys were suspended in HBSS with collagenase type I 0.4 $\mathrm{mg} / \mathrm{mL}$ (Sigma \#C0130), collagenase type IV $1 \mathrm{mg} / \mathrm{mL}$ (Sigma \#5138), DNAase I $10 \mu \mathrm{g} / \mathrm{mL}$ (Sigma \#DN25), and 1\% BSA (Sigma \#7409). After thoroughly mincing the tissue and digesting for 30 min at $37^{\circ} \mathrm{C}$ at $150 \mathrm{rpm}$ in a shaking water bath, the cells were filtered through a $70-\mu \mathrm{m}$ cell strainer (BD Biosciences). Following washing steps, the cells were harvested, resuspended in DMEM, and filtered through a $40-\mu \mathrm{m}$ cell strainer (BD Biosciences). Cells were treated with Cytofix/Cytoperm buffer (BD Biosciences \#554714) and stained with PE rat anti-mouse CD326 (BD Bioscience \#563477) to gate epithelial cell population; FITC BrdU flow kit (BD Biosciences \#559619) was used to identify cell frequencies at stages of the cell cycle. Data were acquired with LSRII flow cytometry.

\section{Statistical Analysis}

Numeric data were shown as means \pm SEMs, significance of differences was assessed by one-way ANOVA or $t$ test whichever is appropriate. Kaplan-Meier curve differences were determined using the log-rank test. Data analyses and graphing were performed using GraphPad Prism v8 (GraphPad Software), $p<0.05$ was considered statistically significant.

\section{Results}

\section{Anti-TIMP-2 Increases Epithelial Cell Cycle Progression}

To understand the role that TIMP-2 may play in the early phase of septic AKI, a neutralizing antibody was administrated to the CLP-sepsis mice. TIMP-2 in the whole kidney (Fig. 1a, b) but especially in tubule lumen (Fig. 1c, d) was significantly inhibited in the animals treated with the TIMP-2 antibody. With no substantial effects on $\mathrm{CD} 45^{+}$renal inflammatory cells (data not presented), 


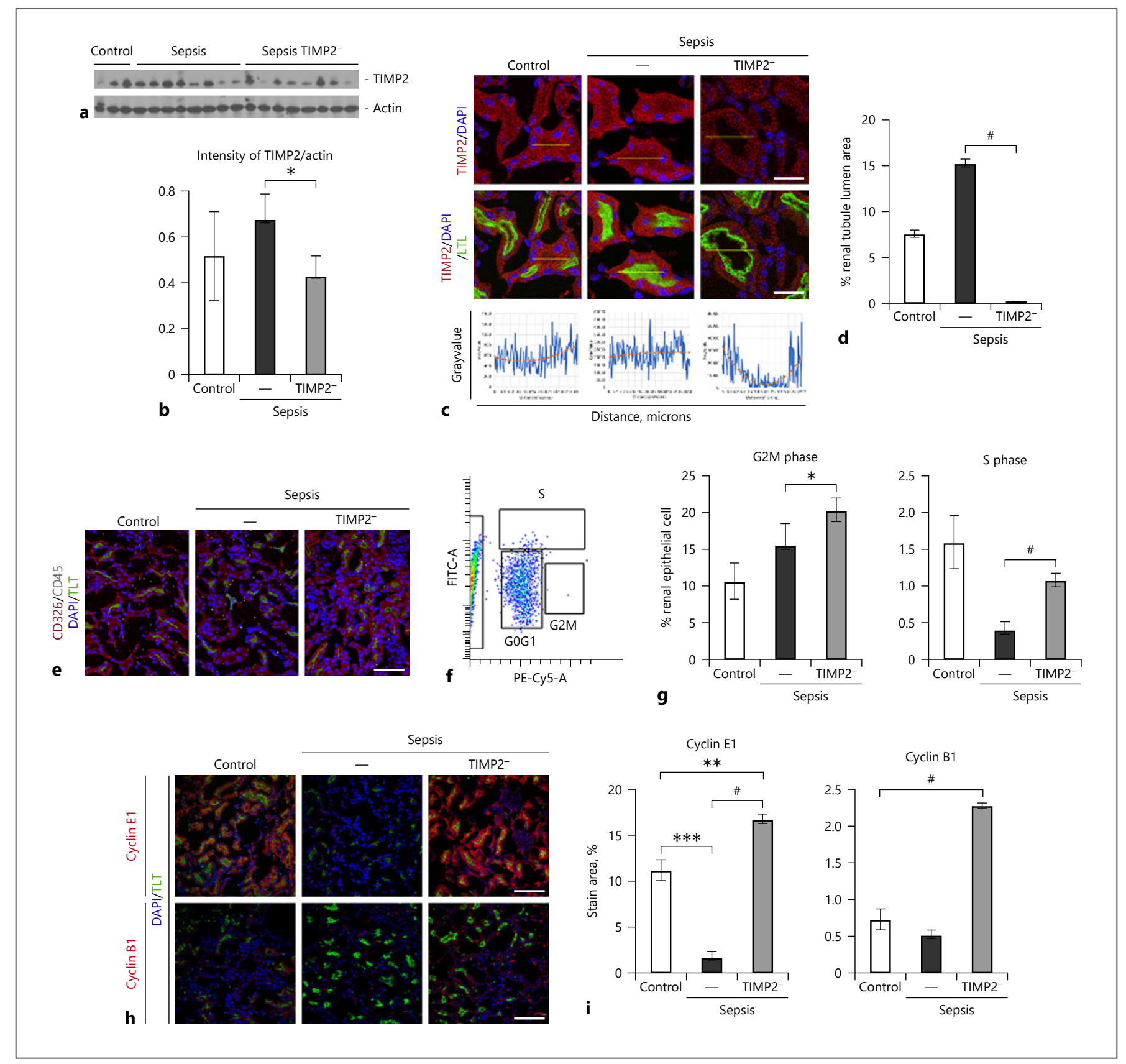

Fig. 1. Secreted TIMP-2 modulates renal cell cycle progression. Murine sepsis was induced by CLP surgery. Renal expression of TIMP-2 and the proportions of epithelial cell in cell cycle phases were measured in a murine model of anti-TIMP-2 treated sepsis. a, b Blots of renal TIMP-2 and the corresponding quantified intensities relative to actin expression. Statistical significance between treated and untreated septic animals was examined by $t$ test. c Representative images of TIMP-2 immunofluorescence stain with the plots showing below the pixel intensities and their trend lines of renal TIMP-2 across the tubule lumen denoted by the yellow line in the tissue images. Scale bar $=30 \mu \mathrm{m}$. d Quantified TIMP-2 intensities in the tubule lumen obtained from 3 samples/group and 5 fields/per sample. Statistical significance between treated and un- treated septic animals was examined by $t$ test. e, f Representative images of $\mathrm{CD}^{2} 26^{+}$cell loci in the kidneys and the flow cytometry gated renal epithelial cell (by CD326 ${ }^{+}$) to determine cell frequencies at phases of G0/G1, G2/M, and S. Scale bar $=60 \mu \mathrm{m}$. g The corresponding group quantifications of cell frequencies at phases of G2/M and S. $n=6$ for each group. Statistical significance was examined by $t$ test. $\mathbf{h}$ Representative images of cyclin E1 and cyclin B1 immunofluorescence stain. Scale bar $=60 \mu \mathrm{m}$. i Quantified cyclin E1 and cyclin B1 stain area in the kidneys obtained from 3 samples/group and 5 fields/per sample. Statistical significance was examined by ANOVA. ${ }^{*} p<0.05 ;{ }^{* *} p<0.01 ;{ }^{* * *} p<0.001 ;{ }^{*} p<$ 0.0001 . TIMP-2, tissue inhibitor of metalloproteinases-2; CLP, cecal ligation and puncture; LTL, Lotus tetragonolobus lectin. 


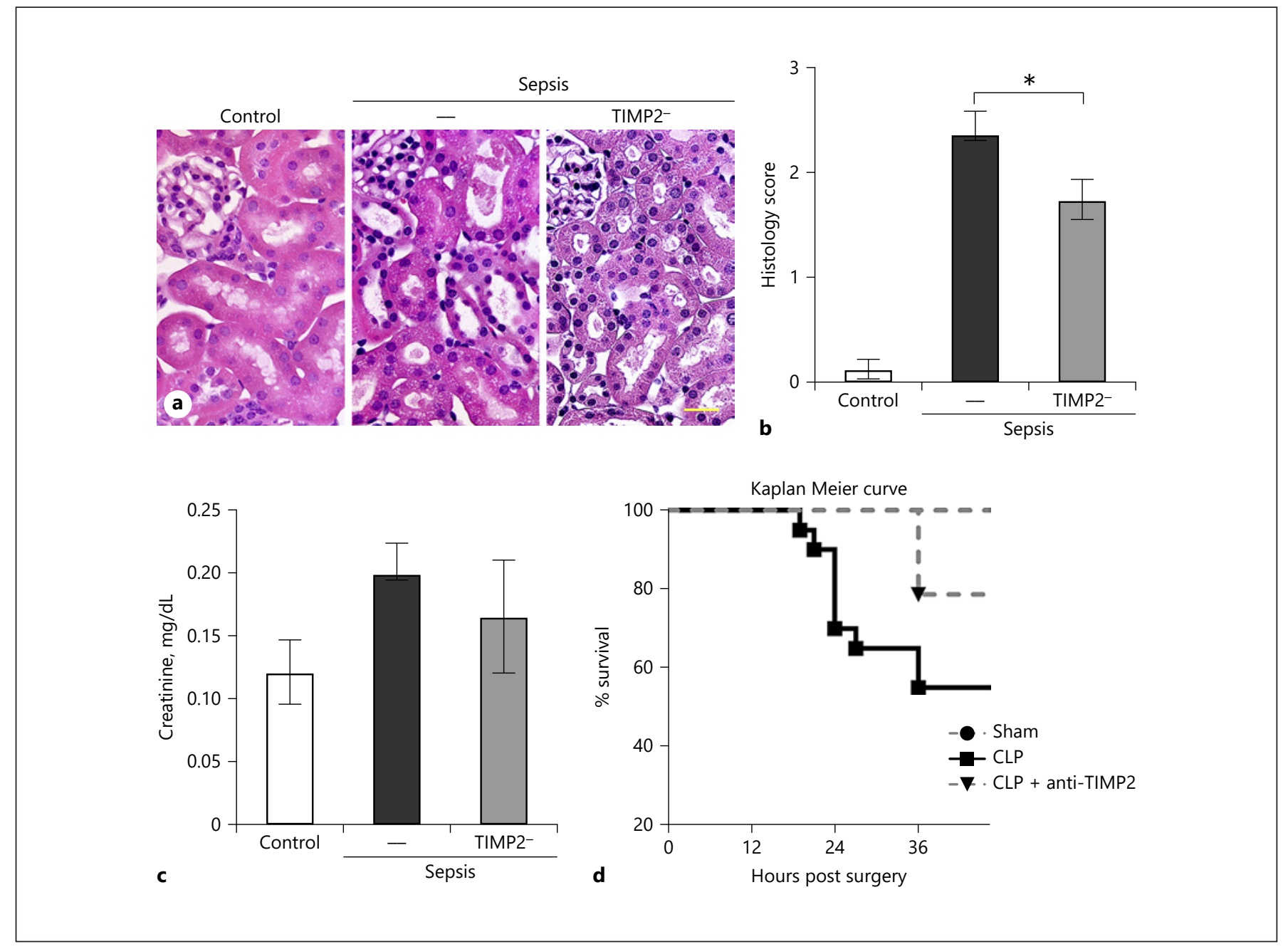

Fig. 2. Murine sepsis was induced by CLP surgery. a Representative images of renal H\&E stain. Scale bar $=30 \mu \mathrm{m}$. $\mathbf{b}$ Renal tubule injury scores obtained from 3 samples/group and 5 fields/per sample. Statistical significance between treated and untreated septic animals was examined by $t$ test. c Statistical significance of SCr level was examined by $t$ test. d Kaplan-Meier survival curves for group animals. Survival $n=8$ for each group. Difference of survival rates was analyzed using log-rank Mantel-Cox test. ${ }^{*} p<0.05$. CLP, cecal ligation and puncture; H\&E, hematoxylin and eosin; SCr, serum Cr. treatment significantly increased the proportion of renal epithelial cell progressing into G2/M and S phases (Fig. 1e, g) compared to septic controls, with cell loci confirmed by immune stain and gated in flow by $\mathrm{CD}_{2} 26^{+}$. Kidney cells in septic animals had lower levels of cyclin E1; TIMP2 inhibition significantly increased cyclin $\mathrm{E} 1$ and $\mathrm{B} 1$ in the kidneys, which is essential for G1/S and G2/M cell cycle progression (Fig. 1h, i). These results show that by inhibiting TIMP-2 in renal tubule cells, sepsis-induced cell cycle arrest can be partially reversed.

\section{TIMP-2-Mediated Renal Injury during Sepsis}

Renal histologic damage and inflammation were examined in both sham- and CLP-treated animals, with or without treatment with a TIMP-2-neutralizing antibody. Sepsis produced characteristic renal tissue damage with tubular epithelial cell vacuolization. However, animals treated with anti-TIMP-2 had significantly reduced tissue injury yet had a mild impact on kidney function and survival rates compared with septic controls (Fig. 2a-d). These results demonstrate that blocking TIMP-2 could ameliorate renal histologic injury in a sepsis model. 


\section{Discussion/Conclusion}

Despite its utility as a biomarker for AKI, the role of TIMP-2 in the pathogenesis of AKI has not been well explored. Both clinical and experimental studies have reported increased urinary TIMP-2 level hours before/during AKI $[3,5,7,8]$. However, whether such expression is pathologic or adaptive has not been clarified. Given that cell cycle arrest may be adaptive or maladaptive depending on the duration and phase of the cycle [12], it is likely that TIMP-2 expression may be adaptive or maladaptive depending on clinical context. Given the possibility that the injured cells may undergo phenotype revision and dedifferentiation back to more primitive states, these cells may secrete TIMP-2 which appears to function as a growth factor involved in kidney morphogenesis and epithelial cell branching [13]. Thus, the effects of TIMP-2 may vary depending on the cell types involved.

This is now the second study to demonstrate that despite the protective roles of TIMP-2, inhibition of this molecule can be beneficial in models of S-AKI. Our study extends the work of Li et al. [9] by demonstrating that cell cycle is directly affected by TIMP-2 inhibition as well as showing beneficial effects of targeting TIMP-2 in a more realistic model of sepsis. Finally, our intervention involved antibodies directed against TIMP-2 which represents an easier commercialization pathway than siRNA knockdown. Our study design was based on the consensus that antibody fragments are filtered and reabsorbed in the renal tubule, and their local maximum concentration usually lasts for days [14].

There are additional known functions of TIMP-2, including suppression of matrix metalloproteinase (MMP) activity. In addition, it is also a pluripotent factor that affects cell apoptosis, proliferation, and differentiation either directly through cell surface receptors or indirectly through modulation of protease activity [10]. Specific to its role in kidney injury, TIMP-2 is reported to promote harmful signaling cascades through activating MMP in the non-inflammatory model, activating NF- $\mathrm{kB}$ signaling, and promoting apoptosis in inflammatory models of AKI $[9,15]$. Consistent with these reports, our study found treatment of TIMP-2 antibody ameliorated kidney tissue injury in S-AKI. The presumed mechanism includes not only the well-known suppressing of MMP activity but also a direct effect of renal epithelial cell cycle arrest. Importantly, we did not appreciate improved kidney function with anti-TIMP-2. Sepsis is known to impair kidney function in multiple ways, and discordance between function and damage has been well described. In conclusion, our study confirmed TIMP-2 not only acts as a marker but is also an active player in S-AKI and a potentially important drug target.

\section{Acknowledgements}

We would like to express our very great appreciation to the Flow Cytometry Core Facility, Research Histology Service at the Thomas E. Starzl Transplantation Institute and the Center for Biological Imaging at the University of Pittsburgh for their help with these studies.

\section{Statement of Ethics}

All the procedures were conducted according to the National Institutes of Health Guide for the Care and Use of Laboratory Animals and were approved by the Institutional Animal Care and Use Committee at the University of Pittsburgh (Protocol \#14094486).

\section{Conflict of Interest Statement}

J.A.K. reports consulting fees and grant support from Astute Medical/BioMerieux. In addition, the University of Pittsburgh and Astute Medical have filed a patent on the use of anti-TIMP-2 for treatment of AKI (WO2018081578A1). No other competing interests are declared.

\section{Funding Sources}

This project was supported by the Center for Critical Care Nephrology, University of Pittsburgh, and Astute medical. The contents of this paper are solely the responsibility of the authors.

\section{Author Contributions}

The study was designed and conceived by X. Wen and J.A.K. J.Z., X. Wan, and X. Wen were responsible for the animal experiments and X. Wen for laboratory analyses. X. Wen and J.A.K. were responsible for interpreting the results. $\mathrm{X}$. Wen drafted the manuscript, and all authors agree with this edition of the draft.

References

1 Chertow GM, Burdick E, Honour M, Bonventre JV, Bates DW. Acute kidney injury, mortality, length of stay, and costs in hospitalized patients. J Am Soc Nephrol. 2005; 16(11):3365-70.

2 Kellum JA. Diagnostic criteria for acute kidney injury: present and future. Crit Care Clin. 2015;31(4):621-32. 
3 Bhatraju PK, Zelnick LR, Herting J, Katz R, Mikacenic C, Kosamo S, et al. Identification of acute kidney injury subphenotypes with differing molecular signatures and responses to vasopressin therapy. Am J Respir Crit Care Med. 2019;199(7):863-72.

4 Russell JA, Walley KR, Singer J, Gordon AC, Hébert PC, Cooper DJ, et al. Vasopressin versus norepinephrine infusion in patients with septic shock. N Engl J Med. 2008;358(9):87787.

5 Kashani K, Al-Khafaji A, Ardiles T, Artigas A, Bagshaw SM, Bell M, et al. Discovery and validation of cell cycle arrest biomarkers in human acute kidney injury. Crit Care. 2013; 17(1):R25.

6 Meersch M, Schmidt C, Hoffmeier A, Van Aken $\mathrm{H}$, Wempe C, Gerss J, et al. Prevention of cardiac surgery-associated AKI by implementing the KDIGO guidelines in high risk patients identified by biomarkers: the PrevAKI randomized controlled trial. Intensive Care Med. 2017;43(11):1551.
7 Emlet DR, Pastor-Soler N, Marciszyn A, Wen $\mathrm{X}$, Gomez H, Humphries WH, et al. Insulinlike growth factor binding protein 7 and tissue inhibitor of metalloproteinases-2: differential expression and secretion in human kidney tubule cells. Am J Physiol Renal Physiol. 2017; 312(2):F284-96.

8 Johnson ACM, Zager RA. Mechanisms underlying increased TIMP2 and IGFBP7 urinary excretion in experimental AKI. J Am Soc Nephrol. 2018;29(8):2157-67.

9 Li Y-M, Zhang J, Su L-J, Kellum JA, Peng Z-Y. Downregulation of TIMP2 attenuates sepsisinduced AKI through the NF-kb pathway. Biochim Biophys Acta Mol Basis Dis. 2019; 1865(3):558-69.

10 Seo DW, Li H, Guedez L, Wingfield PT, Diaz T, Salloum R, et al. TIMP-2 mediated inhibition of angiogenesis: an MMP-independent mechanism. Cell. 2003;114(2):171-80.
11 Zarbock A, Schmidt C, Van Aken H, Wempe C, Martens S, Zahn PK, et al. Effect of remote ischemic preconditioning on kidney injury among high-risk patients undergoing cardiac surgery: a randomized clinical trial. JAMA. 2015;313(21):2133-41

12 Bonventre JV. Maladaptive proximal tubule repair: cell cycle arrest. Nephron Clin Pract. 2014;127(1-4):61-4.

13 Barasch J, Yang J, Qiao J, Tempst P, Erdjument-Bromage $\mathrm{H}$, Leung $\mathrm{W}$, et al. Tissue inhibitor of metalloproteinase-2 stimulates mesenchymal growth and regulates epithelial branching during morphogenesis of the rat metanephros. J Clin Invest. 1999;103(9): 1299-307.

14 Ryman JT, Meibohm B. Pharmacokinetics of monoclonal antibodies. CPT Pharmacometrics Syst Pharmacol. 2017;6(9):576-88.

15 Wang Z, Famulski K, Lee J, Das SK, Wang X, Halloran P, et al. TIMP2 and TIMP3 have divergent roles in early renal tubulointerstitial injury. Kidney Int. 2014;85(1):82-93. 